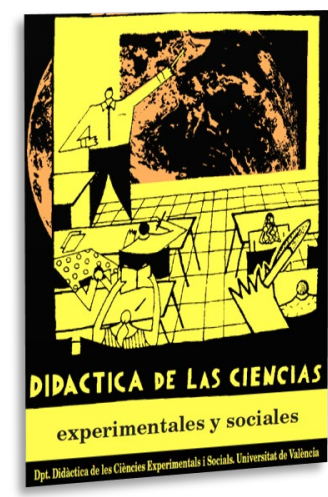

\title{
Ciudadanía y lectura del espacio geográfico en la formación docente de Educación Infantil
}

\author{
Citizenship and reading of the geographical \\ space in pre-service early childhood teacher \\ training
}

DOI: $10.7203 / D C E S .41 .19207$

\author{
Manuel José López Martínez \\ Universidad de Almería, mlm138@ual.es \\ ORCID iD: https://orcid.org/0000-0001-6653-1398
}

\begin{abstract}
RESUMEN: La escuela puede fomentar una lectura crítica y transformadora del espacio geográfico para activar a la ciudadanía infantil en las sociedades democráticas. En esta investigación acción se muestra el proceso de formación seguido con estudiantes de Grado en Educación Infantil. El estudio parte de una pregunta: ¿cómo podemos incrementar la calidad del pensamiento geográfico en el futuro docente novel para fortalecer el derecho de las infancias a la participación ciudadana? Los resultados muestran las posibilidades de realizar un proceso de reflexión progresiva sobre la práctica docente y el pensamiento geográfico en tres contextos: el aula universitaria, el aula escolar de la fase del Practicum y el trabajo de campo realizado en un espacio público urbano. El ejercicio reflexivo en la formación inicial docente sobre el espacio geográfico vivido puede contribuir a la consecución de una mayor justicia social a favor de los y las infantes.
\end{abstract}

Palabras Clave: ciudadanía, infancia, formación docente, espacio geográfico, acción reflexiva

\begin{abstract}
Schools can encourage a critical and transformative reading of geographical space to activate children's citizenship in democratic societies. This action research shows the training process followed with students of the Degree in Early Childhood Education. The study is based on the question: how can we increase the quality of the geographical thinking in future new teachers to strengthen children's right to citizen participation? The results show the possibilities of carrying out a process of progressive reflection on teaching practice and geographical thinking in three contexts: the university classroom, the school classroom during the Practicum phase and the fieldwork carried out in an urban public space. The reflective exercise in initial teacher training on the experienced geographical space may contribute to the achievement of greater social justice for children.
\end{abstract}

KEYWORDS: citizenship, childhood, teacher training, geographical space, reflective action

Fecha de recepción: diciembre de 2020

Fecha de aceptación: febrero de 2021

El contenido de este artículo ha sido posible gracias a la participación del alumnado de $3^{\circ} \mathrm{A}$ y B del Grado en Educación Infantil de la Universidad de Almería que nos ha obligado a reflexionar sobre nuestra práctica docente en la formación inicial en los cursos académicos 2016-17 y 2017-18. Este trabajo forma parte del Proyecto I+D+i "Educación para el futuro y esperanza en la democracia. Repensar la enseñanza de las ciencias sociales en tiempos de cambio (EpF+ED)" (Referencia: PID2019-107383RB-I00), subvencionado por el Ministerio de Ciencia e Innovación (Institución responsable: Universidad Autónoma de Barcelona). 


\section{INTRODUCCIÓN}

La formación inicial ha de incidir en los procesos de pensamiento, creencias y concepciones didácticas de los futuros docentes noveles puesto que en numerosas ocasiones están muy arraigadas antes de decidir ser docente (Porlán, Rivero y Martín del Pozo, 1998). Sus futuras prácticas educativas pueden estar marcadas por creencias configuradas en modelos de enseñanza tradicionales, descriptivos y enciclopédicos si no se les acompaña para que sean analizadas conscientemente. Por ello, dependiendo de cómo se construya el entorno de aprendizaje, así será la toma de conciencia sobre las posibilidades que ofrecen los espacios públicos para la enseñanza del pensamiento espacial y geográfico en Infantil. En los cursos 2016-17 y 2017-18 propusimos al alumnado matriculado en la asignatura anual "Aprendizaje de las Ciencias Sociales en Educación Infantil" del Grado en Educación Infantil (en adelante, EI) que centrara la atención en sus concepciones geográficas implícitas. La finalidad era la de reconocerlas y trasladarlas de manera explícita a otras más complejas y ricas. Esta fue la razón del trabajo que aquí se presenta. En esta investigación cualitativa partimos de la pregunta ¿cómo mejorar la formación inicial del futuro profesorado novel desde un pensamiento geográfico (Araya y Cavalcanti, 2017) alternativo al tradicional, para que redunde en una formación integral de la ciudadanía infantil? Se procede a dar respuesta conociendo que el ayuntamiento de la ciudad donde habitamos, Almería, solicita el reconocimiento como Ciudad Amiga de la Infancia para el período 2018-2022.

Como sostiene Borja (2014) "el test de la ciudad democrática es el espacio público. Y sólo se ejerce la democracia si hay espacio público" (p. 22). Las vivencias en estos espacios son esenciales para favorecer la justicia social y reconocer la diversidad en una sociedad democrática. Sin embargo, existen obstáculos para dar cabida a una participación real de la infancia en la toma de decisiones políticas, a pesar del mandato de la Convención de los Derechos del Niño y de la Niña de 1989. Como plantean Novella y Trilla (2014), "la participación infantil está en boca de todos, pero falta la parte esencial y es que sea real, que forme parte de nuestra cotidianidad y, sobre todo, de la forma de vivir de los niños" (p. 28). Sostenemos que para lograr una verdadera participación y no a un mero simulacro (Hart, 1993), se ha de leer críticamente el espacio público en las escuelas usando un conocimiento geográfico emancipador (Souto, 2007). Por esta razón, interesa fomentar en el futuro docente novel una vivencia alternativa y no sesgada del espacio geográfico para que su alumnado de Infantil retenga aprendizajes que le permitan ejercer el oficio de la ciudadanía (Bárcena, 1997), participando en calidad y cantidad en la toma de decisiones de la comunidad adulta.

Las investigaciones recientes nos dicen que el aprendizaje geográfico adquirido en la etapa preuniversitaria no ha sido el adecuado para responder a los desafíos del presente, al fundamentarse ampliamente en un modelo de enseñanza tradicional, unidireccional y descontextualizado. Pero, en nuestro caso, el problema de fondo reside también en una formación geográfica insuficiente para entender el derecho de los infantes a decidir en y sobre su espacialidad. Esta carencia detectada en cursos anteriores se compartió con los estudiantes universitarios que participaron en la investigación con la intención de analizarla conjuntamente (Elliot, 1990) e incidir en la mejora de su venidera acción docente. En este sentido, entendemos que una de las finalidades de la escuela pública consiste en fomentar una lectura crítica y transformadora del espacio geográfico para promover una intervención real de la ciudadanía infantil en su medio social. Ayuste y Trilla (2020) reconocen que el aula y las instituciones educativas tienen la oportunidad de convertirse en espacios públicos de deliberación racional y de reflexión crítica sobre las cuestiones de poder. Para que esto ocurra, la clave está en diseñar y tejer aprendizajes contextualizados en la formación inicial con el fin de fortalecer a un profesorado reflexivo, ampliando su nivel de conciencia espacial y geográfica, capaz de ofrecer soluciones educativas al comprobar la desigualdad entre adultos e infantes a la hora de vivir, sentir y habitar el espacio público. 


\section{MARCO TEÓRICO Y ESTADO DE LA CUESTIÓN}

Partimos del conocimiento del movimiento de ciudades educadoras iniciado en Barcelona en los años 90, de las experiencias educativas sobre participación ciudadana desarrolladas en Europa, como es el caso del proyecto STEP («Pedagogia della cittadinanza e formazione degli insegnanti: un'alleanza tra scuola e territorio») con profesorado de infantil y primaria en Italia, Francia y España (Estepa y García Pérez, 2020), de las experiencias de la ciudad de Sevilla (De Alba, García Pérez y Santisteban, 2015; García Pérez y De Alba, 2007; Moreno-Fernández y García Pérez, 2015), de prácticas innovadoras en Portugal y España en el Proyecto "Nosotros Proponemos" iniciado en 2011 (Rodríguez y Claudino, 2018), y del análisis detallado de La ciudad de los niños de Tonucci (2009).

Desde este marco teórico, la inmersión en un pensamiento geográfico alternativo se adquiere desmontando una representación de la geografía como conocimiento escolar obsoleto e inútil en el proceso formativo del alumnado universitario (Fernández Caso, 2008; García Monteagudo, Morote y Souto 2019). Esa idea se ha ido cristalizando en el imaginario social a pesar de las transformaciones sufridas por la epistemología geográfica en la segunda mitad del siglo XX (Olcina, 2018). Como sostiene De la Calle (2013), "la forma de entender la materia..., supone que se extienda esta visión popular de lo que es la ciencia geográfica, alejada de los presupuestos de una enseñanza de la geografía más problematizadora y crítica con nuestro entorno social" (p.35). En esta línea, Souto (2011) resalta esa identificación popular de los contenidos geográficos con contenidos enciclopédicos al quedar también recogidos en el currículo educativo. Hoy, las relaciones espaciales son más contradictorias, precisando la ciudadanía claves para interpretar una red de flujos propios de la sociedad de la información (Appadurai, 2001; Castells, 1989). La finalidad educativa está, pues, en poner luz en el binomio confusión/opacidad consustancial a un mundo globalizado, reflejo de unas relaciones de poder desiguales que no se aprecian con facilidad en cualquier espacio geográfico. Por tanto, es prioritario generar el debate sobre cómo y para qué introducir el conocimiento espacial y geográfico en EI. En esta línea, se están desarrollando esfuerzos investigadores y formativos innovadores que demuestran que sí es posible pensar geográficamente en la etapa de Infantil (Canet, Morales y García, 2018; Morales, Giménez, Caurín y Souto, 2020). También tomamos en cuenta, por un lado, la contribución del movimiento de la práctica docente reflexiva (Sáiz y Susinos, 2017) y por otro, el campo de las children's geographies (Malatesta y Granados, 2017). Pero el eje teórico principal de nuestra investigación está basado en el reconocimiento de las aportaciones de la infancia para ofrecer soluciones a problemas de la sociedad actual. Como afirman Castro, Ezquerra y Argos (2016) la investigación educativa ha recogido en la última década estudios que involucran de forma activa, voluntaria y consciente a los niños, adoptándose la perspectiva infantil en el marco de un proceso participativo en el que infantes y adultos se convierten en co-intérpretes de la realidad que experimentan, mitigándose de este modo el dominio adultocéntrico sobre la realidad educativa objeto de estudio.

Por otra parte, una de las consecuencias del modelo económico capitalista ha sido la expansión privada del hábitat urbano. De ahí la necesidad de repensar los espacios públicos desde el punto de vista educativo y político (Colodro y Cadierno, 2017). Souto (2007) afirma que "la utilidad del conocimiento geográfico estriba en que sea capaz de emancipar el conocimiento escolar (de alumnos y profesores) de las rutinas enciclopédicas y culturalistas que aleja este saber escolar de las preocupaciones cotidianas" (p.23). Sin embargo, somos conscientes de sus dificultades (Liceras, 2000, 2016; García Ruíz y Jiménez, 2006) pues el alumnado universitario no ha adquirido una base disciplinar específica ajustada a los contextos actuales, como demuestran Marrón, (2011), Buzo e Ibarra (2014), Benimelis y Ordinas (2016), Martínez (2017) y De Miguel (2018). En el caso concreto de nuestro estudiantado, se da la paradoja de que su contacto con las dimensiones epistemológicas, metodológicas, curriculares y prácticas de la geografía escolar se produce sólo en tercer curso de dicho Grado, tras una experiencia poco gratificante con esta materia en la etapa preuniversitaria. Esta investigación educativa se ha ido fraguando en dos años, donde se ha podido analizar la evolución de 
cada grupo. Hemos de propiciar una adecuada formación en el profesorado para "guiar al alumnado hacia cuestiones relevantes del espacio vital significativo, como es el medio próximo" (Morales, Giménez, Caurín y Souto, 2020, p. 35). En cuanto a la investigación sobre la enseñanza de la geografía en EI se han de mencionar las aportaciones de Catling (1993), Martin y Owens (2005), Massey (2005). En los niveles universitarios de nuestro país aquella ocupa un lugar reducido si se compara con su presencia en las etapas de Secundaria y Primaria, si bien contamos con contribuciones como las de Canet, Morales y García (2018), Morales y Santana (2019) y las de Canet, Morales y Santana (2018), estas últimas sobre la incidencia de las representaciones sociales en la formación docente. Sin embargo, Sebastiá y Tonda (2014) han detectado que los profesionales de la geografía en la universidad están más preocupados por la investigación en la propia ciencia que en la forma de enseñar. Por su parte, Marrón (2011) critica la escasez de la formación geográfica en el magisterio de Infantil y Primaria en la Universidad Complutense con la incorporación al EEES, y Martínez (2017) denuncia el arrinconamiento disciplinar que sufre la geografía en los nuevos Grados de Educación.

En este marco es relevante investigar sobre la práctica de la enseñanza de las Ciencias Sociales (en adelante, CC.SS.) en los contextos y en las aulas universitarias (Pagès, 2011), pues la formación superior debe impactar en el futuro docente para que éste compruebe la situación de injusticia social que se vive hoy en cuanto al grado de participación de la infancia, por ejemplo, en la toma de decisiones en la organización espacial. Dicho lo anterior, una de las finalidades educativas de la universidad ha de orientarse hacia la consecución de mayores cotas de igualdad en el espacio geográfico, teniendo en cuenta cómo los niños viven la ciudad y cómo son las relaciones de poder entre estos y los planificadores adultos de los espacios públicos. Pensamos que es necesario, desde la función docente, dar un decidido paso cultural y político para fortalecer la democracia real en un escenario de intermediación donde el profesorado ha de estar presente realizando lecturas críticas del espacio, teniendo en cuenta las distintas escalas vinculando el conocimiento geográfico, el escolar y el profesional con el cotidiano o experiencial de su alumnado (García Pérez, 2015).

La cuestión está en cómo hacer este trayecto formativo en un curso académico. Porlán y Rivero (1998) advertían que este no puede sustentarse "en la yuxtaposición aditiva de contenidos formativos, por más que esos contenidos puedan ser potencialmente muy interesantes desde la perspectiva del formador" (p.146). Siguiendo lo anterior, en el curso 2016-17 reorientamos nuestra estrategia de enseñanza de las CC.SS. en el Grado de EI con la finalidad de incrementar el uso educativo de los conocimientos geográficos sobre los espacios vividos, percibidos y concebidos (Souto, 2018) en el alumnado del Grado, para que acompañe en un futuro próximo a sus infantes a la hora de vivir y sentir la ciudadanía participando (Rodríguez y Claudino, 2018) en la configuración y mejora de los espacios colectivos.

\section{OBJETIVOS Y METODOLOGÍA}

Esta transformación en la estrategia formativa se originó después de haber observado carencias en el aprendizaje de la enseñanza de la geografía en años anteriores. Estas estaban motivadas por una resistencia a la hora de tratar el espacio geográfico en EI desde el enfoque de la geografía crítica y transformadora. A partir de aquí, se inició un ejercicio reflexivo conjunto sobre la práctica docente, aprovechando situaciones reales. En un principio, fueron dos las acciones innovadoras incorporadas. Una, invitando al alumnado en formación a la realización de una micro-investigación etnográfica individual cuyo fin era conocer cómo se trabaja el espacio geográfico en el aula de la EI en la fase del Practicum. Otra, propiciando un análisis de un parque público para ser aprovechado como recurso educativo desde los planteamientos de una geografía de la percepción, crítica y humanística.

El objetivo principal de la investigación consiste en llevar a cabo un proceso formativo para que el futuro profesorado novel promueva en la escuela lecturas reflexivas transformadoras del espacio geográfico para fortalecer el oficio de la ciudadanía infantil. A tenor de lo dicho, es obvio que la configuración de una práctica docente precisa de una atención pausada cuando se manejan 
conocimientos novedosos. Dicha atención pasa por distintas fases, tal y como se puede observar en el Cuadro 2. Además, como demuestra la investigación reciente (Novella et al., 2014) con un mayor reconocimiento real de la participación ciudadana desde edades tempranas, se obtendrá una mayor calidad democrática. Por este motivo, los infantes pueden convertirse en actores sociales emergentes si son acompañados por sus maestras y maestros en la institución educativa, realizando valoraciones sobre la calidad del espacio vivido y aportando soluciones a los problemas revelados en él. Con este objetivo, pretendemos que el futuro profesorado estimule una alfabetización científica (Blanco et al., 2008) en el aula de EI, contribuyendo así a la construcción colectiva del espacio público, siendo prioritario para ello que el adulto-docente fomente el derecho a cambiar y a reinventar la ciudad (Harvey, 2005) desde un enfoque de igualdad y equidad. Por esta razón, invitamos al alumnado universitario a colaborar en esta investigación acción como agente de transformación que puede contribuir a reducir las desigualdades intergeneracionales y la injusticia en la sociedad (Zeichnner, 2010).

Para atender el objetivo propuesto, seleccionamos la metodología de la investigación acción puesto que "es un poderoso instrumento para reconstruir las prácticas y los discursos sociales" (Latorre, 2014, p. 372). Se trata, como indican Kemis y McTaggart (1988) de un proceso construido desde y para la práctica, con el fin de mejorarla y comprenderla, demandando una actuación grupal y colectiva en las distintas fases de la investigación, generando un análisis crítico constante en las diferentes situaciones. La estrategia en espiral de la actuación investigadora se activó con el tratamiento en el aula universitaria de dos metaconceptos: el tiempo histórico y el espacio geográfico, ejes interconectados para una formación integral de los y las infantes, presentes de forma implícita en las tres áreas de conocimiento del desarrollo curricular vigente tanto en el territorio estatal como en el autonómico de Andalucía (Real Decreto 1630/2006, Decreto 428/2008 y Orden del 5 de agosto de 2008). Prosiguió con dos hitos destacados, por una parte, un trabajo de campo en los colegios del Practicum II para familiarizarlo con la investigación educativa, y continuó con un análisis geográfico e interpretativo del parque de Las Familias ${ }^{1}$ de la ciudad de Almería como recurso didáctico. Previamente se había formulado una serie de preguntas-eje en clase para fusionar el binomio teoríapráctica, diagnosticándose la situación de partida. Estas preguntas fueron: ¿cuáles son las finalidades y los objetivos del trabajo docente con el conocimiento geográfico en esta etapa educativa? ¿Cuáles son las claves de la enseñanza de la geografía en un mundo globalizado donde predomina el espacio urbano? ¿Qué entornos de aprendizaje se podrían utilizar para mejorar el proceso de enseñanzaaprendizaje del espacio geográfico y fortalecer una ciudadanía infantil democrática? ¿Qué modelo de docente se tendría que experimentar para aprovechar al máximo el conocimiento geográfico escolar en estas edades iniciales? Estas cuestiones se reformularon en las 3 preguntas de nuestra investigación acción, planteando si el futuro profesorado novel:

1. ¿Conoce en profundidad las finalidades de la enseñanza de las CCSS y de la geografía escolar hoy? ¿Cómo podemos familiarizarnos con ellas?

2. ¿Posee y maneja las claves suficientes para enseñar geografía sabiendo que nos encontramos en un mundo globalizado donde predomina el espacio urbanizado? ¿Cómo podemos hacer que las consiga?

3. ¿Puede acompañar al alumnado de la etapa de Infantil para que participe de forma real en la toma de decisiones en su espacio público, cumpliendo así el mandato de la

\footnotetext{
${ }^{1}$ Este parque está ubicado en una parte del antiguo recinto ferial colindando con uno de los ejes vertebradores que transcurre de norte a sur en esta ciudad andaluza, la avenida del Mediterráneo. Es muy frecuentado por la ciudadanía local y muy próximo a una zona residencial en expansión hacia la antigua huerta denominada Vega de Acá que mira hacia levante y al río Andarax. Fue seleccionado debido a las posibilidades didácticas que presenta para la educación Infantil y para realizar un análisis espacial desde una orientación crítica de la geografía. Se estructura en 4 subespacios cargados de simbolismo identitario simulando la Alcazaba de Almería, el Parque Natural Cabo de Gata-Nijar, Almería Tierra de Cine y una zona vinculada al Reciclaje de residuos.
} 
Convención de los Derechos del Niño y de la Niña de 1989 y ampliando la calidad de la Democracia? ¿Cómo debe hacerse este acompañamiento?

Estos interrogantes nos invitan a pensar qué y cómo hacemos para que el conocimiento geográfico sea asimilado a lo largo de un curso académico. Ante este desafío, la metodología cualitativa permite explorar caminos posibles para detectar el currículo retenido desde el punto de vista subjetivo, usando distintas técnicas e instrumentos de recogida de información a lo largo de esta asignatura anual entre las que destacamos: cuestionario abierto para registrar ideas previas, elaboración de diagnósticos usando la matriz DAFO, observación participante y no participante, entrevistas semiestructuradas, grupos de discusión en el aula, diario personal o portafolio, trabajos colaborativos elaborando mapas conceptuales, diseño de itinerarios y de unidades didácticas integradas, análisis y discusión de diversos documentos personales y públicos. Desde aquí, realizando un ejercicio permanente de triangulación con todo lo anterior y a partir de unas relaciones pedagógicas simétricas (Sáiz y Susinos, 2017) se diseñaron sucesivos entornos de aprendizaje que se reorientaron en el curso 2017-18 (véase Cuadro 2, Fase $3^{\mathrm{a}}$ ). Para abordar este reto se planificaron tres contextos formativos para contrastar los aprendizajes adquiridos: el proceso de enseñanza y aprendizaje de las CC.SS. en el aula universitaria, la fase del Practicum II en las aulas de los colegios y la salida de campo al parque público de Las Familias ${ }^{2}$. Este espacio fue seleccionado como recurso y medio para realizar in situ un análisis e interpretación desde una orientación crítica de la geografía y desde la óptica infantil.

Las preguntas de investigación nos acompañaron en la realización de sucesivas tareas entre este formador y el alumnado, quedando recogidas sus respuestas en portafolios individuales, en microinvestigaciones etnográficas realizadas en el Practicum y en cuadernos de campo. Estas han servido como fuentes de información para llevar a cabo esta investigación, para alentar en el estudiante la toma de conciencia y apropiación "de sus ideas y modelos mentales, pues nadie aprende si no se adueña de su propio conocimiento, reconociéndose a sí mismo como sujeto epistémico" (Rivero y Porlán, 2017, p. 74). En este marco de referencia, la hipótesis de progresión (Porlán y Rivero, 1998) nos ha permitido interpretar la realidad e intervenir en ella orientando el proceso formativo, teniendo en cuenta el nivel deseable que debía alcanzar el alumnado en esta asignatura. Este marco incluía 6 secciones (ver Cuadro 1).

CUADRO 1. Pautas seguidas en las fases de la investigación acción y mapa de categorización (en negrita) para el análisis de la información obtenida

\begin{tabular}{cl}
\hline Secciones & $\begin{array}{l}\text { Pautas de investigación acción y mapa } \\
\text { de categorizaciones (en negrita) }\end{array}$ \\
\hline & $\begin{array}{l}\text { Que asimile e interiorice mediante una } \\
\text { práctica reflexiva continuada, la } \\
\text { presencia imprescindible de las } \\
\text { dimensiones espacio-temporales en el } \\
\text { conocimiento de la realidad social }\end{array}$
\end{tabular}

Que conozca y tenga en cuenta las finalidades de las CC.SS. en el marco

Sección 2 escolar, especialmente la que consolida una formación ciudadana activa en un escenario globalizado

\footnotetext{
${ }^{2}$ Véase: http://mejoralmeria.es/parque-de-las-familias. Artículo publicado el 7 febrero, 2015 por Dani Plaza (actualizado el 30 junio, 2017)
} 


\begin{tabular}{|c|c|}
\hline Sección 3 & $\begin{array}{l}\text { Que mida hasta dónde llega el peso de la } \\
\text { tradición en sus concepciones y } \\
\text { creencias a la hora de concebir para qué } \\
\text { sirve el pensamiento geográfico }\end{array}$ \\
\hline Sección 4 & $\begin{array}{l}\text { Que valore ampliamente que cualquier } \\
\text { espacio es un medio de comunicación. } \\
\text { Este ofrece una información rica para ser } \\
\text { leída usando para ello principios, } \\
\text { conceptos, habilidades } \\
\text { procedimientos geográficos básicos } \\
\text { para formar a la ciudadanía infantil }\end{array}$ \\
\hline Sección 5 & $\begin{array}{l}\text { Que compruebe las posibilidades } \\
\text { educativas que aporta el aprendizaje de } \\
\text { pensar el espacio geográfico para } \\
\text { impulsar un adecuado desarrollo de las } \\
\text { diferentes capacidades contempladas en } \\
\text { el currículo de EI }\end{array}$ \\
\hline Sección 6 & $\begin{array}{l}\text { Que conciba el espacio como una } \\
\text { construcción dinámica y plural donde } \\
\text { intervienen distintos elementos y } \\
\text { factores, naturales y humanos que } \\
\text { pueden incrementar o reducir las } \\
\text { desigualdades socioespaciales entre } \\
\text { adultos e infantes }\end{array}$ \\
\hline
\end{tabular}

Fuente: elaboración propia

\section{Proceso de inVestigación y Resultados}

El programa formativo expuesto forma parte del trabajo con cuatro grupos de tercero del Grado en EI, dos en el curso 2016-17 y dos en el curso 2017-18, con 245 estudiantes con presencia regular en clase, oscilando cada grupo entre 60-65, representando el $90 \%$ mujeres y el $10 \%$ hombres, de un total de 263 matriculados oficialmente sumando los cursos 2016-17 (130) y 2017-18 (133) en la asignatura anual obligatoria "Aprendizaje de las Ciencias Sociales en Educación Infantil" con 9 ECTS. La muestra, de tipo no probabilístico por conveniencia, está representada por el alumnado de $3^{\circ}$ del Grado. Su perfil formativo era el siguiente: 75\% estudió Bachillerato, $15 \%$ un ciclo superior de Formación Profesional, 8\% otras titulaciones y 2\% pruebas de acceso para mayores de 25 años.

En el Cuadro 2 se muestra de forma resumida el cronograma, los contenidos formativos y las acciones investigadoras a lo largo del curso académico 2016-17, incluyendo una novedad en el curso 2017-18, reflejo de un diseño flexible propio de cualquier investigación-acción. Partíamos de las ideas del alumnado sobre sus vivencias y creencias relativas al conocimiento geográfico (Sandín, 2003; Tójar, 2006) detectadas con un diagnóstico de la situación, continuando con un plan abierto de acción, su puesta en práctica y finalizando con la fase de resultados. 
CUADRO 2. Plan de acción. Cronograma, contenidos formativos, acciones investigadoras a lo largo del curso 2016-17

\section{Cronograma}

\begin{abstract}
Fase1 ${ }^{a}$ diagnóstica
Desde septiembre hasta noviembre
\end{abstract}

\section{Contenidos formativos}

Conceptos claves históricos y geográficos fundamentales + epistemología de la historia y de las diversas geografías + el conocimiento histórico y geográfico escolar + planteamientos teóricos $\mathrm{y}$ prácticos innovadores en EI

Practicum II en un centro educativo. Realización de una micro-investigación

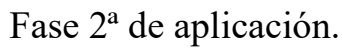

Noviembre, diciembre, enero etnográfica sobre el tratamiento del tiempo y del espacio en el aula de infantil

\section{Acciones, técnicas e instrumentos de investigación}

-Comprobación del grado de formación en la didáctica específica de la geografía mediante cuestionario de respuesta abierta.

-Completa el cuestionario el $80 \%$ del alumnado matriculado, analizándose los resultados en clase.

-Observación participante

-Elaboración de una plantilla de registro semanal sobre cómo se lleva a cabo la enseñanza y el aprendizaje del tiempo y del espacio en las aulas de los ciclos de infantil

-Elaboración de un Informe final de una investigación cualitativa etnográfica entregada a la vuelta del Practicum II -Análisis de contenido de cada informe individual y feedback evaluativo

-Diseño en grupos de trabajo de un itinerario didáctico por el parque de Las Familias pensado para alumnado de segundo ciclo de Infantil.

El parque de Las Familias: un laboratorio para practicar lo aprendido sobre las CC.SS., como disciplinas científicas y como conocimiento escolar.

Fase $3^{\text {a }}$ aplicación. Desde marzo, abril, mayo
En el curso 2017-18, como novedad, el alumnado participa en EIDA ${ }^{3} 2018$ dando a conocer sus microinvestigaciones etnográficas a la comunidad académica de la UAL (Universidad de Almería)

-Análisis de contenido del diseño grupal de un itinerario

-Elaboración en grupo de un informe conjunto a partir de los informes individuales realizados en el Practicum II sobre el tratamiento del tiempo y del espacio en las aulas de Infantil

-Creación de grupos de discusión

-Invitación a la participación grupal en EIDA para exponer los resultados obtenidos en las micro-investigaciones al contrastar los informes individuales de cada componente del grupo

-Grupo de discusión y observación participante

Desvelar los cambios epistemológicos que se producen durante el proceso formativo + solución a problemas conectados con la práctica docente a partir de supuestos prácticos $\mathrm{o}$ simulaciones
-En la elaboración de las comunicaciones grupales que se presentaban a EIDA, se compartían mediante grupo de discusión los aprendizajes alcanzados durante todo el proceso formativo de la asignatura, apreciándose debilidades y fortalezas

Fuente: elaboración propia

3 III Encuentro de Investigación del Alumnado (EIDA), 30 de mayo de 2018. Véase: https://cms.ual.es/UAL/universidad/departamentos/educacion/noticias/IIIEIDA2018 
Centrándonos en los resultados obtenidos, si tenemos en cuenta la información recabada al inicio del curso mediante un cuestionario de respuesta abierta sobre diversos contenidos, comprobamos que la mayor parte del alumnado (85\%) no tenía una formación básica en la didáctica específica de la geografía. No diferenciaba entre espacio y territorio, no poseía un conocimiento real sobre la utilidad de la ciencia geográfica hoy y no sabía cómo se podría emplear el conocimiento geográfico escolar en EI mediante la imagen-huella del niño Aylan Kurdi. Vistos los resultados, a lo largo del primer cuatrimestre tuvo que familiarizarse con el uso de los conceptos clave de las CC.SS. (Benejam, 1999) y con los metaconceptos de tiempo y espacio.

A partir de los objetivos establecidos y con el propósito de dar voz a los participantes, aportamos una selección de fragmentos representativos que emergieron en los diferentes contextos de intercambio, diálogo y discusión con el alumnado (cuestionario inicial, entrevistas, grupos de discusión, observación participante, análisis de contenido de informes individuales) evidenciándose así los significados que los participantes atribuían a la realidad formativa. Presentamos unos marcos explicativos que recogen el sentir de la mayor parte del estudiantado, en los que se aprecian los cambios experimentados desde el inicio hasta el final de la investigación acción, vinculándose con las diferentes secciones del mapa de categorización (en negrita) incluido en el Cuadro 1. En la primera fase comprobamos cuál era el modelo mayoritario de enseñanza y aprendizaje de las CC.SS. en la etapa preuniversitaria. Veamos el caso siguiente:

Como futura docente, me interesé por investigar qué asignaturas iba a tener en tercero (...) y cuando vi que teníamos una asignatura llamada Aprendizaje de las ciencias sociales en Educación Infantil, me llevó a pensar cómo me iría, ya que durante mi infancia no me gustaron las ciencias sociales hegemónicas porque siempre me las han impuesto como algo teórico y que he tenido que aprender de memoria para superar el examen y así poder pasar de curso. Al recordar lo mal que se me dio en el colegio, me cuestioné si sabría enseñarlas como futura docente. (Alumna G3 de $3^{\circ} \mathrm{A}$, noviembre de 2017, reflexión portafolio). Secciones detectadas del Cuadro 1: 3, 5.

Si describimos el recorrido en espiral propio de la investigación acción, diremos que, en una primera fase, el alumnado asimila cuestiones relacionadas con los contenidos epistemológicos de la historia y de las diversas geografías, con la hegemonía de una representación social de estas disciplinas, con nociones y conceptos históricos y geográficos claves, así como con principios, metodologías y estrategias a usar en el proceso de enseñanza. Fue preciso que en esta fase valorara los recursos didácticos aprovechables en el entorno, detectara las dificultades de aprendizaje de la historia y de la geografía en EI y se familiarizara con propuestas prácticas y teóricas innovadoras y tradicionales sobre el conocimiento social escolar (Trepat y Comes, 2002; Cooper, 2002; Egan, 2007; Miralles y Rivero, 2012; Aranda, 2016).

Como se aprecia en el Cuadro 2, en las fases $2^{\mathrm{a}}$ y $3^{\mathrm{a}}$ de aplicación, el alumnado se adentra en una micro-investigación educativa cualitativa de carácter etnográfico desde el campo de la didáctica de las CC.SS. Para ello, aprovechando la fase de Practicum II, elabora individualmente para esta asignatura un informe final donde recoge la indagación, descripción e interpretación sobre el tratamiento del tiempo histórico y del espacio geográfico en el aula de infantil, contrastando lo madurado en el primer cuatrimestre del curso. En esta fase se presenta el siguiente marco explicativo debido a la riqueza de su contenido al resaltar el potencial aprovechamiento didáctico de un espacio vivido y percibido por niños y niñas de segundo ciclo de infantil, su patio de recreo, vinculándolo con el principio de temporalidad, aspecto primordial en esta etapa educativa. Fue recogido en el informe final que realizó una alumna en un CEIP de un municipio del poniente almeriense.

Conclusión personal.

(...), he de destacar la importancia de los espacios y del tiempo en la práctica educativa diaria. Muchos maestros/as consideran que el único espacio de aprendizaje es el aula y el único momento 
que deben conocer es el presente y esto no es así. Existen más lugares y maneras de conseguir que nuestros niños/as aprendan, pero los maestros/as se limitan. Por ejemplo, el patio es un espacio al que apenas se le da importancia para aprender y puede servir para realizar descubrimientos mediante juegos, como yincanas o excavaciones, teatros donde interactúen, se pongan en el lugar de los demás... Incluso, puede utilizarse para hacer un museo o exposiciones sobre vestidos, comidas, herramientas de distintos espacios del mundo, ya que el colegio es un espacio de encuentro de culturas. (Informe de alumna G2 de $3^{\circ} \mathrm{A}$ entre el 15 de febrero y el 7 de abril de 2017). Secciones detectadas del Cuadro 1: 1, 3, 4, 5, 6.

Seguidamente, y antes de iniciar la visita al parque de Las Familias ${ }^{4}$, en el segundo cuatrimestre del curso se debatió en el aula universitaria el estado de la cuestión sobre la participación ciudadana infantil en la toma de decisiones en los espacios públicos, estudiando las aportaciones de Tonucci (2009) y de Novella et al. (2014). Es significativo el grado de sensibilización alcanzado por los y las estudiantes sobre el problema de las vivencias de los infantes en los espacios urbanos. Aportamos tres reflexiones que demuestran los efectos de la participación en la investigación para fortalecer la labor docente:

Hoy hemos visitado el parque de Las Familias, situado en la zona este de Almería. Con esta salida pretendemos conocer si realmente las ciudades y, concretamente los espacios que se supone que están destinados para los niños y niñas, cumplen con los requisitos adecuados y van a servir para satisfacer sus deseos, necesidades o intereses, o si simplemente se construyó como un elemento más para contribuir a la estética de la ciudad. (...) Tras un análisis de las distintas zonas que componen el recinto, determinando sus debilidades y fortalezas, debemos realizar una programación (...) para convertirla en un itinerario didáctico con alumnado de segundo ciclo. (Alumna $\mathrm{G} 3$ de $3^{\circ} \mathrm{B}$, mayo de 2018, reflexión de portafolio sobre la visita al parque Las Familias). Secciones detectadas del Cuadro 1: 2, 4, 5, 6 .

Una de las preguntas planteadas en un grupo de discusión (GD) fue: ¿cómo os habéis sentido en este trabajo de observación y de análisis geográfico del parque? Las respuestas contribuyen a entender las influencias del trabajo de campo realizado en este parque.

Alumna MG: Sinceramente, después de pasar casi todos los días por este parque, me he dado cuenta de la cantidad de detalles que se pueden aprovechar desde la geografía para enseñarlos. Hasta ahora no me había dado cuenta de todos ellos. Con las actividades relacionadas con las ciencias sociales que hemos realizado (...), puedo aprovecharlas como futura maestra. (GD 2 de $3^{\circ} \mathrm{B}$, mayo de 2018, sobre la salida de campo al parque Las Familias). Secciones detectadas del Cuadro 1: 4, 5 .

Alumna ES: (...) Una vez que lo estudiamos con calma y despacio, se puede apreciar que en algunos espacios no se ha tenido en cuenta a los niños y niñas de infantil para que desarrollen todas sus capacidades. Incluso veo algunas zonas que son peligrosas (...). Pero veo también oportunidades para que aprendan sobre el tiempo histórico y el espacio geográfico, además de los problemas medioambientales actuales y sobre el cuidado hacia el patrimonio. (GD 2 de $3^{\circ} \mathrm{B}$, mayo de 2018, sobre la salida de campo al parque Las Familias). Secciones detectas del Cuadro 1: 1, 3, $4,5,6$.

Sabemos que la planificación de cualquier investigación acción puede sufrir cambios en su desarrollo. Así, en el curso académico 2017-18 incorporamos una novedad que consistió en dar a conocer públicamente la investigación realizada por el alumnado en su fase de Practicum II en un foro creado por el Departamento de Educación en la Universidad de Almería. Propusimos a los grupos de trabajo de la asignatura la participación en los Encuentros de Investigación del Alumnado (EIDA)

\footnotetext{
${ }^{4}$ Véase enlaces a Google Maps: https://goo.gl/maps/khreLvHLGPapNk6s6 y https://goo.gl/maps/Aav1k9DSiGjb5hsC9
} 
2018, con el fin de que mostraran a la comunidad universitaria el conocimiento obtenido con sus micro-investigaciones sobre el tratamiento del espacio y del tiempo en EI en los centros educativos de la provincia de Almería durante su fase de Practicum. De esta manera, y más allá de las fronteras del espacio áulico de la asignatura, se reconocía la investigación educativa aportada por el alumnado de formación inicial en una institución académica formal. En algunos casos, esta incursión investigadora del alumnado quedó recogida en una publicación académica (Lirola et al., 2019). Con esta estrategia, contribuíamos a que el o la estudiante del Grado tomara conciencia de las posibilidades de transferencia de conocimiento de sus aportaciones al campo de la investigación en la enseñanza y aprendizaje de las ciencias sociales en EI.

Con respecto a la fase final del proceso formativo, reproducimos dos reflexiones representativas por el significado de su contenido. Estas nos permiten calibrar los cambios (aprendizajes) experimentados de forma mayoritaria por el alumnado en cuanto a la conexión entre teoría-práctica, práctica-teoría, desarrollo de destrezas y resolución de problemas enlazando el pensamiento geográfico e histórico.

Durante toda mi vida escolar entendí las CC.SS. como una asignatura poco cercana, donde sólo exigían tener buena memoria para aprobar un examen (valor de cambio). Ahora ha cambiado mi visión, ya que a través de ellas podemos hacer ver y entender a nuestro alumnado lo importante que son los cambios en la historia y en el espacio, las formas de pensar, vivir y el valor que como seres sociales e individuales tenemos. Como docente utilizaré el modelo crítico para acercar a mis niños/as a estas ciencias... (Alumna G8 de $3^{\circ} \mathrm{A}$, junio de 2018). Secciones detectadas del Cuadro $1: 1,2,3,4,5,6$.

Después de haber estado estudiando la asignatura, tengo un concepto más amplio de lo que son las CC.SS. y de cómo debo enseñarles a mis futuros alumnos/as los conceptos de tiempo y espacio, ya que, si consigo formar personas capaces de pensar histórica y espacialmente, estaré cumpliendo con mi objetivo, que es formar ciudadanos justos, libres, autónomos, competentes, críticos, cívicos, comprometidos y responsables con nuestro mundo. (...) Hay que creer más en los niños y niñas y darles voz para que opinen y participen en la vida social y política, ya que ellos también forman parte de la sociedad. (Alumna G7 de $3^{\circ} \mathrm{B}$, junio de 2018). Secciones detectadas del Cuadro 1: 1, 2, 3, 4, 5, 6 .

Los resultados obtenidos muestran que, si propiciamos una reflexión progresiva sobre la práctica docente centrada en la revisión de su pensamiento geográfico, estaremos más cerca de alcanzar una mayor justicia social incrementando la participación infantil en la toma de decisiones en todos los asuntos que le afecten, cumpliendo así las exigencias políticas establecidas en la Convención de los Derechos del Niño y de la Niña de 1989.

\section{DISCUSIÓN Y CONCLUSIONES}

En los fragmentos seleccionados se desvelan los cambios percibidos en el proceso formativo del alumnado al participar en la investigación-acción (Elliot, 1990). Han aflorado las teorías implícitas (Pozo, 2016) que el estudiante llevaba consigo sobre el pensamiento geográfico, para aproximarlas a otro más crítico y creativo desde la posición de las infancias, desmontándose una representación de la geografía (Fernández Caso, 2008; García Monteagudo, Morote y Souto, 2019) como conocimiento obsoleto e inútil. Recordemos que esta era una meta que pretendíamos alcanzar. Teniendo presentes las finalidades y el objetivo de esta investigación, el proceso de enseñanza reflexiva sobre la práctica docente defendido por Sáiz y Susinos (2017) supone un reto como formadores universitarios no exento de limitaciones. Estas se han superado mediante estrategias orientadas a remover sentimientos, emociones y conocimientos vinculados a la identidad profesional y a sus saberes. Por ello, el trabajo de campo, tanto en el Practicum II como en un parque público 
urbano, se han convertido en hitos de aprendizaje, centrándonos en tres aspectos básicos para favorecer una formación inicial reflexiva sobre el pensamiento geográfico.

En el primero, trasladamos al alumnado universitario la relevancia de construir su identidad docente para afrontar como argumentaba Zeichnner (2010) las desigualdades de la sociedad actual, insistiendo en la urgencia de consolidar su profesión como agente de transformación social, apoyándonos en la práctica e investigando sobre ella. Así, se ponen en valor tanto las experiencias de las micro-investigaciones etnográficas difundidas públicamente en EIDA 2018 como el trabajo de campo en el parque Las Familias. Sirviéndonos de estas oportunidades de aprendizaje realizamos una labor de reconocimiento para demostrar que los estudiantes son sujetos epistémicos (Rivero y Porlán, 2017) capaces de elaborar un nuevo conocimiento, revisando sus concepciones y creencias sesgadas sobre el pensamiento geográfico tradicional procedente de etapas preuniversitarias.

En el segundo, comprueban los efectos positivos de enseñar CC.SS. en EI, puesto que contribuyen al desarrollo de todas las capacidades de los infantes. En este tiempo, hemos analizado desde el punto de vista teórico y práctico cómo podemos aprovechar educativamente el pensamiento geográfico, para lo cual hemos usado diferentes giros de la geografía (Lindón e Hiernaux, 2010). Nos acercamos así a unos contenidos epistemológicos más actualizados de esta ciencia, siendo conscientes de que educamos a un futuro profesorado generalista con unos itinerarios de aprendizaje que dependen de los contextos de formación vividos.

En el tercer aspecto se pone el acento en la necesidad de convertirnos en docentes que pueden intervenir en las estructuras y problemáticas sociales que se reflejan en los espacios geográficos. Desde esa perspectiva, hemos hecho hincapié en una de las finalidades de las ciencias sociales: la política. Como se ha podido apreciar en el tercer marco explicativo mediante una lectura del parque de Las Familias, los futuros docentes pueden fortalecer la educación integral de su alumnado de EI fomentando un conocimiento geográfico emancipador (Souto, 2007), contribuyendo a su formación como ciudadano participativo, crítico y comprometido con la solución de los problemas de su tiempo, para mejorar la calidad del oficio de la ciudadanía que Bárcena (1997) otorga a la educación y que Novella y Trilla (2014) reclaman, para que la participación infantil sea efectiva en el espacio público, contribuyendo así al fortalecimiento de la democracia (Borja, 2014).

Como formadores, creemos que estos esfuerzos reflexivos precisan de una aplicación efectiva en la escuela y su correspondiente evaluación, bien a través de un seguimiento en las prácticas externas, en los trabajos finales de estudio o bien en los primeros años como docentes. Por tanto, aunque esta formación inicial no garantiza al cien por cien el uso de un saber geográfico crítico en cualquier contexto educativo, sí ha sido una experiencia relevante para sopesar las posibilidades de dicha orientación. Por último, comprobamos que la formación inicial mejora cuando convertimos la lectura crítica del espacio público en una acción política-educativa para reforzar tanto a los futuros docentes como al derecho de la ciudadanía infantil a participar en la toma de decisiones en el espacio público.

\section{Referencias}

Appadurai, A. (2001). La modernidad desbordada. Dimensiones culturales de la globalización. Buenos Aires: FCE.

Aranda, A. M. (2016). Didáctica de las ciencias sociales en educación infantil. Madrid: Síntesis.

Araya, F. y Cavalcanti, L. de Souza (2017). Construcción del pensamiento geográfico en estudiantes de Licenciatura en Geografía de la Universidad Federal de Goias (UFG). Didáctica Geográfica, (18), 23-37.

Ayuste, A. y Trilla, J. (2020). Un sexto principio para el manifiesto por una pedagogía post-crítica. Teoría de la Educación, 32(2), 35-26. 
Bárcena, F. (1997). El oficio de la ciudadanía. Introducción a la educación política. Barcelona: Paidós.

Benejam, P. (1999). La oportunidad de identificar conceptos clave que guíen la propuesta curricular de las ciencias sociales. Íber. Didáctica de las Ciencias Sociales, Geografia e Historia, 21, 512.

Binimelis, J. y Ordinas, A. (2016). Los conocimientos mínimos de Geografía en los estudios de Gado en Educación Primaria. Revista Complutense de Educación, 27(3), 1309-1326.

Blanco, P., De La Calle, M., Fernández, C., Molero, B., Ortega, D. y Sánchez Agustí, M. (2008). La formación en didáctica de las Ciencias Sociales del maestro de educación infantil. Una propuesta adaptada al Espacio Europeo de Educación Superior. En Ávila, Rosa María, Cruz, Alcázar y Díez, Ma Consuelo (Eds.), Didáctica de las Ciencias Sociales, Currículo Escolar y Formación del Profesorado. La didáctica de las Ciencias Sociales en los nuevos planes de estudio. Jaén: Universidad de Jaén, AUSPDCS, UNIA.

Borja, J. (2014). Prólogo del libro de Diego Sánchez y Luis Ángel Domínguez (Coords.), Identidad y espacio público. Ampliando ámbitos y prácticas. Barcelona: Gedisa Editorial.

Buzo, I. e Ibarra, P. (2014). La posición de la Geografía en la Educación Secundaria y el Bachillerato. Madrid: AGE. Recuperado de: http://ciug.cesga.es/PDF/informeage13.pdf.

Canet, S, Morales, A. J. y García, D. (2018). Pensar geográficamente en la educación infantil: de la imaginación a la construcción social del espacio concebido. Didáctica Geográfica, (19), 23-46.

Canet, S, Morales, A.J. y Santana, D. T. (2018). Repensar las representaciones escolares desde la participación activa: estudio de caso en el segundo ciclo de educación infantil. En V. Peris, D. Parra y X.M. Souto (Coords.), Repensamos la geografia e historia para la educación democrática (pp. 89-102). Valencia: Nau Llibres.

Castells, M. (1989). La ciudad informacional. Tecnologías de la Información, reestructuración económica y proceso urbano-regional. Madrid: Alianza.

Castro, A, Ezquerra, P. y Argos, J. (2016) Procesos de escucha y participación de los niños en el marco de la educación infantil: Una revisión de la investigación. Educación XX1, 19(2), 105126. DOI: $10.5944 /$ educXX1.14271.

Catling, S. (1993). The whole world in our hands. Geography, 78(4), 340-358.

Colodro, J. U. y Cadierno, J. (2017). El sistema de espacios públicos en un ámbito metropolitano complejo: el caso del Gran Santiago. Anales de Geografia de la Universidad Complutense, 37(1), 67-85. DOI: 10.5209/AGUC.55956.

Cooper, H. (2002). Didáctica de la historia en la educación infantil y primaria. Madrid: Morata.

De Alba, N., García Pérez, F. y Santisteban, A. (Coord.) (2015). Educar para la participación ciudadana en la enseñanza de las Ciencias Sociales. Sevilla: Asociación Universitaria de Profesores de Didáctica de las Ciencias Sociales y Díada Editora.

De La Calle, M. (2013). La enseñanza de la geografía ante los nuevos desafíos medioambientales, sociales y territoriales. En R. De Miguel, M ${ }^{\mathrm{a}}$ L. De Lázaro y Torres, M J. Marrón Gaite (Coords.), Innovación en la enseñanza de la geografía ante los desafios sociales y territoriales (pp. 33-52). Zaragoza: Institución Fernando El Católico.

De Miguel, R. (2018). Geografía y tiempo contemporáneo: educación geográfica y enseñanza de las ciencias sociales para el mundo global. REIDICS, 2, 36-54. DOI: 10.17398/2531-0968.02.36.

Egan, K. (2007). Fantasía e imaginación: su poder en la enseñanza. Una alternativa a la enseñanza y aprendizaje en Educación Infantil y Primaria. Madrid: Morata y Ministerio de Educación y Ciencia.

Elliot, J. (1990). La investigación-acción en educación. Madrid: Morata.

Estepa, J. y García Pérez, F.F. (2020). El modelo del profesor investigador en el aula en el Proyecto IRES. İber. Didáctica de las Ciencias Sociales, Geografía e Historia, 100, 22-28. 
Fernández, M.V. (2008). Formación docente continua y procesos de transformación curricular. Aporte para pensar el cambio y la innovación en la enseñanza de la geografía. Enseñanza de las Ciencias Sociales. Revista de investigación, 7, 99-107.

García Monteagudo, D., Morote, A.F. y Souto, X.M. (2019). Las representaciones del saber académico: aportaciones desde la geografía escolar. Arxius de Ciències Socials, 41, 11-28.

García Ruíz, A. y Jiménez, J. A. (2006). Los principios científico-didácticos (PCD). Nuevo modelo para la enseñanza de la Geografía y de la Historia. Granada: Editorial Universidad de Granada.

García Pérez, F. y De Alba, N. (2007). Educar en la participación como eje de una educación ciudadana. Reflexiones y experiencias. Didáctica Geográfica, 9, 243-258.

García Pérez, F. (2015). El conocimiento escolar en el centro del debate didáctico. Reflexiones desde la perspectiva docente. Con-Ciencia Social, 19, 49-62.

Harvey, D. (2005). A brief history of neoliberalism. Oxford: Oxford University Press.

Hart, Roger A. (1993). La participación de los niños: de la participación simbólica a la participación auténtica, Innocenti Essay, 4, International Child Development Centre.

Kemmis, S. y McTaggart, R. (1988). Cómo planificar la investigación-acción. Barcelona: Laertes.

Latorre, A. (2014). La investigación acción. En R. Bisquerra (Coord.), Metodología de la investigación educativa (pp. 369-394). Madrid: La Muralla.

Liceras, Á. (2000). Las dificultades en el aprendizaje de las Ciencias Sociales y su consideración en el currículo. Revista de Ciencias de la Educación, 182, 171-186.

Liceras, Á. (2016). Las dificultades en la enseñanza y el aprendizaje de las Ciencias Sociales. En A. Liceras y G. Romero (Coords.), Didáctica de las Ciencias Sociales. Fundamentos, contextos y propuestas (pp. 95-115). Madrid: Pirámide.

Lindón, A. y Hiernaux, D. (2010). Los giros de la Geografia Humana. Desafios y horizontes. Barcelona: Anthropos Editorial.

Lirola, M.J., Asensio, M., García, T., López, M.J., Roith, Ch. y Prados, E. (2019). Investigar la educación: Encuentro de Investigación del Alumnado 2018. Almería: Universidad de Almería.

Malatesta, S. y Granados, J. (2018). La contribución de la geografía de los niños y de las niñas a la enseñanza de la geografía. Documents d'Anàlisi Geogràfica, 63(3), 631-640.

Marrón, $\mathrm{M}^{\mathrm{a}} \mathrm{J}$. (2011). Educación geográfica y formación del profesorado. Desafíos y perspectivas en el nuevo espacio europeo de educación superior (EEES). Boletín de la Asociación de Geógrafos Españoles, 57, 313-341.

Martin, F., y Owens, P. (2005). Young children making sense of the world. En S. Scoffham (Ed.), Primary geography handbook (pp. 63-73). Sheffield: Geographical Association.

Martínez, L. C. (2017). La geografía en los estudios universitarios de Magisterio: evolución histórica, situación actual y significado docente. Biblio3W Revista Bibliográfica de Geografía y Ciencias Sociales, XXII, 1207, 1-33.

Massey, D. (2005). For space. Londres: Sage Publications.

Miralles, P. y Rivero, P. (2012). Propuestas de innovación para la enseñanza de la historia en Educación Infantil. REIFOP, 15(1), 81-90.

Morales, A.J., y Santana, D. (2019). Las representaciones de la didáctica de las ciencias sociales en los futuros docentes de educación infantil. En D. Parra y C. Fuertes (Coords.), Reinterpretar la tradición, transformar las prácticas: Ciencias sociales para una educación crítica (pp. 99121). Valencia: Tirant lo Blanch.

Morales, A. J., Giménez, T., Caurín, C. y Souto, X.M. (2020). Educación ambiental y conocimiento del entorno en la etapa de infantil. Aula de Infantil, 104, 33-36.

Moreno-Fernández, O. y García-Pérez, F. (2015). Ciudadanía, participación y compromiso con los problemas socio-ambientales. Concepciones del alumnado participante en programas educativos andaluces. Investigación en la Escuela, 86, 21-34.

Novella, A. M., Llena, A., Noguera, E., Gómez, M., Morata, T., Trilla, J., Agud, I. y Cifre-Mas, J. (2014). Participación infantil y construcción de la ciudadanía. Barcelona: Graó. 
Novella, A. M. y Trilla, J. (2014). La participación infantil. En A. M. Novella et al, Participación infantil y construcción de la ciudadanía (pp. 13-28). Barcelona: Graó.

Olcina, J. (2018). La geografía debe ser -siempre-crítica. Texto presentado en el acto de inauguración del XV Coloquio Internacional de Geocrítica, celebrado en la Facultad de Geografía e Historia de la Universidad de Barcelona, el 7 de mayo de 2018. Recuperado de: http://www.ub.edu/geocrit/XV-Coloquio/JorgeOlcina.pdf.

Pagès, J. (2011). Las relaciones entre investigación y práctica docente en la enseñanza de la historia. En R. López Facal, L. Velasco, V. Santidrián y X. Armas (coords.), Pensar históricamente en tiempos de globalización (pp. 123-146). Santiago de Compostela: Universidade de Santiago de Compostela.

Porlán, R, Rivero, A. y Martín del Pozo, R. (1998). Conocimiento profesional y epistemología de los profesores I: Teoría, métodos e instrumentos. Enseñanza de las Ciencias, 15(2), 155-171.

Porlán, R. y Rivero, A. (1998). El conocimiento de los profesores. Sevilla: Díada.

Pozo, J. I. (2016). Aprender en tiempos revueltos. La nueva ciencia del aprendizaje. Madrid: Alianza Editorial.

Rivero, A. y Porlán, R. (2017). La evaluación en la enseñanza universitaria. En R. Porlán (Coord.). Enseñanza universitaria. Cómo mejorarla. Madrid: Morata.

Rodríguez, M.A. y Claudino, S. (Coords.) (2018). ¡Nosotros proponemos! Ciudadanía, sostenibilidad e innovación geográfica ante los desafios educativos de la sociedad. Barcelona: Graó.

Sáiz Linares, Á. y Susinos, T. (2017). "Nos Dabas la Confianza para Hablar". El Supervisor Universitario en un Practicum Reflexivo. REICE. Revista Iberoamericana sobre Calidad, Eficacia y Cambio en Educación, 15(1), 55-69. DOI: 10.15366/reice2017.15.1.004.

Sandín, M. P. (2003). Investigación cualitativa en Educación. Fundamentos y tradiciones. Madrid: McGraw-Hill.

Sebastiá, R. y Tonda, E. (2014). Líneas de investigación e innovación en la enseñanza de la Geografía, a partir de la revista Scripta Nova. Ar@cne Revista Electrónica de Recursos en internet sobre geografia y ciencias sociales, 186, 1-26.

Souto, X. M. (2007). Educación geográfica y ciudadanía. Didáctica Geográfica, 9, 11-31.

Souto, X.M. (2011). Una educación Geográfica para el siglo XXI: aprender competencias para ser ciudadano en el Mundo Global. Revista Anekúmene, 1, 28-47.

Souto, X.M. (2018). La geografía escolar: deseos institucionales y vivencias de aula. Boletín de la Asociación de Geógrafos Españoles, 79(2757), 1-31. DOI: 10.21138/bage.2757

Tójar, J. C. (2006). Investigación cualitativa. Comprender y actual. Madrid: La Muralla.

Tonucci, F. (2009). ¿Se puede enseñar la participación? ¿Se puede enseñar la democracia? Investigación en la Escuela, 68, 11-24.

Trepat, C. y Comes, P. (2002). El tiempo y el espacio en la didáctica de las ciencias sociales. Barcelona: Graó.

Zeichnner, K. (2010). La formación del profesorado y la lucha por la justicia social. Madrid: Morata.

CÓMO CITAR ESTE ARTÍ́CULO

López Martínez, M. J. (2021). Ciudadanía y lectura del espacio geográfico en la formación docente de Educación Infantil. Didáctica de las ciencias experimentales y sociales, 41, 3-18. DOI: 10.7203/DCES.41.19207. 
